\title{
ANÁLISIS GENÉTICO UNIVARIADO DE CUATRO ENSAYOS DE PROGENIE Y PROCEDENCIA DE Eucalyptus globulus Labill EN CHILE. POTENCIAL GENÉTICO Y SILVÍCOLA
}

\author{
Ipinza, Roberto ${ }^{1}$; Gutiérrez, Braulio² y Molina María Paz ${ }^{3}$
}

\section{RESÚMEN}

Se analiza cuatro ensayos de progenies de Eucalyptus globulus establecidos en Chile, entre las Regiones del Maule y Los Ríos ( $34^{\circ}$ a $40^{\circ}$ LS), que comprenden en promedio 35 procedencias y 224 familias.

El análisis genético corresponde a un análisis univariado para cada ensayo y para cada rasgo; altura y diámetro. Todos los componentes de varianza, heredabilidades y errores estándar asociados se estimaron mediante REML (Restricted Maximun Likelihood).

Se determina los componentes de varianza a través de un modelo mixto de árbol individual y se determina el parámetro genético heredabilidad para las variables altura y diámetro con su respectivo error estándar.

Durante todo el período de evaluación (2 a 83 meses) se observan tendencias crecientes en la varianzas fenotípica y genética aditiva. Esta última aumenta en menor proporción que la primera, siendo significativas las diferencias entre ambas al final de los 67 a 83 meses.

Para la altura el rango observado de valores de heredabilidad fluctúa entre 0,10 a 0,25. Para el diámetro los valores son superiores, fluctuando entre 0,10 a 0,28 . Para ambos rangos se excluyó el ensayo de Tanumé por ser significativamente más joven que los otros ensayos.

Se analiza la estructura genética de la altura y diámetro en función de los componentes de varianza, se establece recomendaciones para cada ensayo y finalmente se discute el potencial genético y silvícola de la especie E. globulus

Palabras clave: Eucalyptus globulus, parámetros genéticos, heredabilidad

\footnotetext{
${ }^{1}$ Dr. Ingeniero de Montes, Sede Los Ríos, Instituto Forestal. Chile. roberto.ipinza@infor.cl

2 Ingeniero Forestal, Sede Bio Bio, Instituto Forestal. Chile. bgutierr@infor.cl

${ }^{3}$ Ingeniero Forestal MSc. Sede Bio Bio, Instituto Forestal. Chile. mmolina@infor.cl
} 


\section{SUMMARY}

Four Eucalyptus globulus progeny tests, comprising an average of 35 provenances and 224 families, established in Chile between the regions of Maule and Los Ríos (34ํㅜ to $40^{\circ} \mathrm{SL}$ ) were analyzed.

Genetic analysis corresponds to a univariate analysis for each test and for each trait; height and diameter. All variance components, heritabilities and associated standard errors were estimated by REML (Restricted Maximun Likelihood).

Variance components are determined through a mixed model of individual tree. The genetic parameter heritability and its respective standard error were also determined.

Throughout the evaluation period (2-83 months) increasing trends were observed for phenotypical and additive genetical variances. The last one increases less than the first one, with significant differences between them at the end of 67-83 months.

The heritability for height varied from 0.10 to 0.25 . For the diameter the values are higher, ranging from 0.10 to 0.28 . Tanumé test was excluded of this analysis because it was significantly younger than the other trials.

The genetic structure of the height and diameter was analyzed in function of the variance components. Recommendations for each test are done. Finally, the genetic and silvicultural potential of the species E. globulus is discussed.

Keywords: Eucalyptus globulus, genetic parameters, heritability. 


\section{INTRODUCCION}

Eucalyptus globulus Labill. es una especie de alta calidad y rendimiento pulpable (Cotterill y Brolin, 1997), la superficie de plantaciones a nivel mundial alcanza a 1,5 millones de hectáreas (Tibbits, et al, 1997).

En Chile existen ya 306.000 ha de plantaciones con esta especie (INFOR, 1997) y constituye la especie de rápido crecimiento productora de fibra corta de mayor importancia económica.

Su valor pulpable la convierte en la especie más apetecida por los fabricante de papel de escritura de alta calidad, la demanda por este tipo de papel continúa creciendo (Kellison, 1997), por lo que es de esperar que las plantaciones de E. globulus también continúen creciendo.

El programa de mejoramiento genético de esta especie en Chile se inició en forma independiente en dos líneas, la primera corresponde a la de la raza local, la que fue desarrollada para su primer ciclo de mejoramiento por Droppelmann y Delmastro (1988) y la otra línea fue iniciada con un programa de introducción de especies y procedencias por el Instituto Forestal en 1962.

Posteriormente, la fase de introducción de progenie y procedencia por Prado (1988), y el programa de mejora propiamente tal por Infante et al. (1991).

Ambos programas se encuentran finalizando sus respectivos ciclos generacionales. En el corto plazo el segundo programa deberá aportar variabilidad al primero. Una opción de infusión fue ya propuesta por Ipinza et al. (1994).

Los énfasis y matices que se presentan en una estrategia de mejora genética dependen del estado de domesticación en que se encuentra la especie y de la variación genética que existe entre y dentro de sus procedencias. El conocimiento de la heredabilidad de los caracteres comerciales y de la forma en que se distribuye la variación genética son también fundamentales para optimizar las ganancias genéticas por unidad de tiempo (Griffin, 1994).

El presente artículo tiene como objetivo analizar las diferencias de los componentes de varianza en cuatro ensayos de progenie y procedencias y cómo cada uno de ellos varia a diferentes edades. También se estima el parámetro genético heredabilidad y su error estándar. Las estimaciones se realizan mediante el modelo de árbol individual definido por Borralho (1995).

\section{MATERIAL Y MÉTODO}

\section{Colección de Semillas}

La colección de semillas para estos ensayos fue adquirida al Centro de Semillas de $\mathrm{CSIRO}^{4}$, Australia, y corresponde a la colección de Gardiner y Crawford (1988) (Cuadro № 1). Entre los criterios de selección de los árboles se encuentran: tamaño, vigor, forma del fuste, tipo de copa y tipo de rama (Prado y Alvear, 1994). La colección para Chile contempla 224 familias individualizadas en 35 lugares de colección o procedencias.

La colección cubre toda la distribución natural de la especie: sur del Estado de Victoria; las islas del estrecho de Bass y Tasmania (Figura № 1).

\footnotetext{
${ }^{4}$ Commonwealth Scientific and Industrial Research Organization
} 
Cuadro № 1

ANTECEDENTES DE LA COLECCIÓN DE SEMILLAS DE E. globulus DE LOS ENSAYOS DE PROCEDENCIA Y PROGENIE DEL INSTITUTO FORESTAL

\begin{tabular}{|c|c|c|c|c|c|c|c|}
\hline \multirow[b]{2}{*}{ № } & \multirow[b]{2}{*}{ Código } & \multicolumn{2}{|c|}{ Procedencia } & \multirow{2}{*}{$\begin{array}{c}\text { Familias } \\
\qquad\left(\mathbf{N}^{\circ}\right)\end{array}$} & \multirow{2}{*}{$\begin{array}{l}\text { Latitud } \\
\text { (LS) }\end{array}$} & \multirow{2}{*}{$\begin{array}{l}\text { Longitud } \\
\text { (LO) }\end{array}$} & \multirow{2}{*}{$\begin{array}{c}\text { Altitud } \\
\text { (msnm } \\
\text { ) }\end{array}$} \\
\hline & & Lugar & Estado & & & & \\
\hline 1 & 16223 & W Apollo Bay & \multirow{10}{*}{ VIC } & 4 & $38^{\circ} 46^{\prime}$ & $143^{\circ} 32^{\prime}$ & 200 \\
\hline 2 & 16224 & S W Apollo Bay & & 7 & $30^{\circ} 49^{\prime}$ & $143^{\circ} 34^{\prime}$ & 145 \\
\hline 3 & 16240 & Otway State Forest & & 18 & $38^{\circ} 25^{\prime}$ & $143^{\circ} 27^{\prime}$ & 150 \\
\hline 4 & 16319 & Jeeralang North & & 30 & $38^{\circ} 19^{\prime}$ & $146^{\circ} 36^{\prime}$ & 220 \\
\hline 5 & 16398 & Hodgson Hedley & & 6 & $38^{\circ} 38^{\prime}$ & $146^{\circ} 30^{\prime}$ & 20 \\
\hline 6 & 16399 & Wilsons Promontory & & 9 & $39^{\circ} 08^{\prime}$ & $146^{\circ} 25^{\prime}$ & 60 \\
\hline 7 & 16400 & N Toora & & 2 & $38^{\circ} 37^{\prime}$ & $146^{\circ} 21^{\prime}$ & 180 \\
\hline 8 & 16402 & W Kennett River & & 6 & $38^{\circ} 39^{\prime}$ & $143^{\circ} 48^{\prime}$ & 250 \\
\hline 9 & 16406 & NW of Lorne & & 6 & $38^{\circ} 31^{\prime}$ & $143^{\circ} 57^{\prime}$ & 210 \\
\hline 10 & 16407 & W Lorne & & 9 & $38^{\circ} 31^{\prime}$ & $143^{\circ} 56^{\prime}$ & 210 \\
\hline 11 & 16410 & Badgers CK Quarry & \multirow{25}{*}{ TAS } & 9 & $41^{\circ} 59^{\prime}$ & $145^{\circ} 18^{\prime}$ & 120 \\
\hline 12 & 16411 & Binalong Bay & & 7 & $41^{\circ} 16^{\prime}$ & $148^{\circ} 18^{\prime}$ & 120 \\
\hline 13 & 16412 & Little Henty River & & 6 & $41^{\circ} 56^{\prime}$ & $145^{\circ} 12^{\prime}$ & 10 \\
\hline 14 & 16417 & $\mathrm{~N}$ of Cape Barren I. & & 5 & $40^{\circ} 22^{\prime}$ & $148^{\circ} 13^{\prime}$ & 20 \\
\hline 15 & 16419 & NW of Cape Barren I. & & 7 & $40^{\circ} 21^{\prime}$ & $148^{\circ} 07^{\prime}$ & 20 \\
\hline 16 & 16421 & SW of Cape Barren I. & & 4 & $40^{\circ} 26^{\prime}$ & $148^{\circ} 03^{\prime}$ & 40 \\
\hline 17 & 16422 & Macquarie Harbour & & 3 & $42^{\circ} 20^{\prime}$ & $145^{\circ} 20^{\prime}$ & 20 \\
\hline 18 & 16424 & King Island & & 4 & $40^{\circ} 00^{\prime}$ & $144^{\circ} 00^{\prime}$ & 60 \\
\hline 19 & 16425 & S of Flinders I. & & 3 & $40^{\circ} 14^{\prime}$ & $148^{\circ} 08^{\prime}$ & 120 \\
\hline 20 & 16426 & NW of Flinders I. & & 1 & $39^{\circ} 46^{\prime}$ & $147^{\circ} 52^{\prime}$ & 20 \\
\hline 21 & 16427 & $\mathrm{~N}$ of Flinders $\mathrm{I}$. & & 3 & $39^{\circ} 45^{\prime}$ & $147^{\circ} 57^{\prime}$ & 40 \\
\hline 22 & 16429 & Central Flinders I. & & 5 & $39^{\circ} 55^{\prime}$ & $147^{\circ} 57^{\prime}$ & 40 \\
\hline 23 & 16431 & Central Flinders I. & & 8 & $40^{\circ} 02^{\prime}$ & $148^{\circ} 01^{\prime}$ & 190 \\
\hline 24 & 16432 & E Flinders I. & & 1 & $39^{\circ} 59^{\prime}$ & $148^{\circ} 11^{\prime}$ & 60 \\
\hline 25 & 16433 & Central Flinders I. & & 3 & $40^{\circ} 04^{\prime}$ & $148^{\circ} 04^{\prime}$ & 200 \\
\hline 26 & 16434 & S of Flinders $\mathrm{I}$. & & 3 & $40^{\circ} 16^{\prime}$ & $148^{\circ} 10^{\prime}$ & 40 \\
\hline 27 & 16470 & Moogara & & 16 & $42^{\circ} 47^{\prime}$ & $146^{\circ} 55^{\prime}$ & 500 \\
\hline 28 & 16471 & NW Dover & & 5 & $43^{\circ} 16^{\prime}$ & $146^{\circ} 59^{\prime}$ & 190 \\
\hline 29 & 16472 & Ellendale & & 5 & $42^{\circ} 38^{\prime}$ & $146^{\circ} 42^{\prime}$ & 460 \\
\hline 30 & 16473 & New Norfolk & & 4 & $42^{\circ} 43^{\prime}$ & $147^{\circ} 09^{\prime}$ & 300 \\
\hline 31 & 16474 & $\mathrm{~N}$ of ST Marys & & 5 & $41^{\circ} 34^{\prime}$ & $148^{\circ} 12^{\prime}$ & 400 \\
\hline 32 & 16475 & SW of Jericho & & 5 & $42^{\circ} 25^{\prime}$ & $147^{\circ} 16^{\prime}$ & 500 \\
\hline 33 & 16476 & S of Geeveston & & 7 & $43^{\circ} 12^{\prime}$ & $146^{\circ} 54^{\prime}$ & 250 \\
\hline 34 & 16477 & $\mathrm{~N}$ of Geeveston & & 3 & $43^{\circ} 08^{\prime}$ & $146^{\circ} 57^{\prime}$ & 200 \\
\hline 35 & 16478 & Tasman Peninsula & & 5 & $43^{\circ} 04^{\prime}$ & $147^{\circ} 50^{\prime}$ & 20 \\
\hline
\end{tabular}




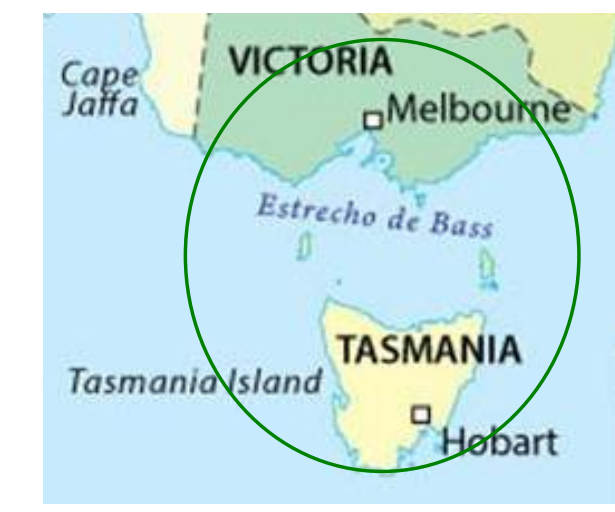

FIGURA № 1

PROCEDENCIAS DE SEMILLA DE Eucalyptus globulus

\section{Individualización de los Ensayos, Diseño y Mediciones}

Los ensayos fueron establecidos entre 1989 y 1991 en cuatro localidades del país (Cuadro № 2). Se utilizó plantas en maceta de uno y dos años en vivero y técnicas de establecimiento intensivas que contemplaron preparación del suelo con subsolador, control de malezas y fertilización inicial.

Como diseño estadístico se utilizó el de bloques de familias compactas, equivalente al de parcelas divididas (split plots) en su distribución en el campo. En este esquema la parcela principal está compuesta por la procedencia y dentro de esta se distribuyen al azar las progenies, representadas por parcelas de cuatro plantas en línea.

Cada ensayo está conformado por 8 a 10 bloques y está rodeado por dos hileras perimetrales de plantas de aislación para eliminar el efecto de borde.

En el análisis genético se excluyó la procedencia Wilsons Promontory, dado que su reducido tamaño sugiere un uso no maderero, por los menos en su forma actual.

\section{Cuadro № 2}

UBICACIÓN DE LOS ENSAYOS CONSIDERADOS EN EL ANÁLISIS

\begin{tabular}{|l|l|c|c|c|c|}
\hline Ensayo & Región y Comuna & Latitud S & Longitud W & Establecimiento & $\begin{array}{c}\text { Progenies } \\
\left(\mathrm{N}^{\circ}\right)\end{array}$ \\
\hline San Agustín & Bio Bio - Cauquenes & $35^{\circ} 57^{\prime}$ & $72^{\circ} 05^{\prime}$ & Jul. 1989 & 224 \\
\hline Los Copihues & Los Ríos - Paillaco & $40^{\circ} 01^{\prime}$ & $73^{\circ} 00^{\prime}$ & Sept. 1989 & 226 \\
\hline Los Hermanos & Bio Bio - Los Alamos & $37^{\circ} 41^{\prime}$ & $73^{\circ} 66^{\prime}$ & Ago. 1989 & 210 \\
\hline Tanumé & O'Higgins - Pichilemu $^{\prime}$ & $34^{\circ} 13^{\prime}$ & $71^{\circ} 54^{\prime}$ & Jun. 1991 & 78 \\
\hline
\end{tabular}

En el Cuadro $\mathrm{N}^{\circ} 3$ se indica las variables evaluadas en cada ensayo y el momento en que se hizo las mediciones. 
VARIABLES EVALUADAS Y OPORTUNIDAD DE LAS MEDICIONES

\begin{tabular}{|c|c|c|c|}
\hline Ensayo & Mes de Control & Año de Control & Variables $\left({ }^{1}\right)$ \\
\hline \multirow{7}{*}{ San Agustín } & Noviembre & 1989 & ALT \\
\hline & Marzo & 1990 & ALT-DAC \\
\hline & Abril & 1991 & ALT-DAC \\
\hline & Mayo & 1993 & ALT-DAP \\
\hline & Junio & 1994 & ALT-DAP \\
\hline & Junio & 1995 & ALT-DAP \\
\hline & Junio & 1996 & ALT-DAP \\
\hline
\end{tabular}

\begin{tabular}{|l|l|c|c|}
\hline \multirow{4}{*}{ Los Copihues } & Noviembre & 1989 & ALT-DAC \\
\cline { 2 - 4 } & Marzo & 1990 & ALT-DAC \\
\cline { 2 - 4 } & Mayo & 1991 & ALT-DAC \\
\cline { 2 - 4 } & Agosto & 1993 & ALT-DAP \\
\cline { 2 - 4 } & Abril & 1995 & ALT-DAP \\
\hline
\end{tabular}

\begin{tabular}{|l|l|c|c|}
\hline \multirow{4}{*}{ Los Hermanos } & Octubre & 1989 & ALT-DAC \\
\cline { 2 - 4 } & Abril & 1990 & ALT-DAC \\
\cline { 2 - 4 } & Marzo & 1991 & ALT-DAC \\
\cline { 2 - 4 } & Abril & 1993 & ALT-DAP \\
\cline { 2 - 4 } & Marzo & 1995 & ALT-DAP \\
\hline
\end{tabular}

\begin{tabular}{|l|l|c|c|}
\hline \multirow{4}{*}{ Tanumé } & Diciembre & 1991 & ALT-DAC \\
\cline { 2 - 4 } & Marzo & 1992 & ALT-DAC \\
\cline { 2 - 4 } & Marzo & 1993 & ALT-DAC \\
\cline { 2 - 4 } & Marzo & 1995 & ALT-DAP \\
\hline
\end{tabular}

(1) $\mathrm{ALT}=$ Altura $(\mathrm{m})$

DAC $=$ Diámetro a la altura del cuello $(\mathrm{cm})$

DAP = Diámetro a la altura del pecho $(\mathrm{cm})$

Las líneas coloreadas destacan que las variables son altura y DAP, igual señal se incluye en todos los cuadros en adelante.

\section{Análisis Estadístico}

Se realizó un análisis univariado por ensayo y edad para las variables consideradas, altura y diámetro. Los registros de la base de datos fueron ordenados por sitio y contienen información para ambas variables.

Fueron eliminados del análisis los árboles muertos, con daños o con más de un fuste. Se utilizó un modelo de árbol individual (animal model) definido por Borralho (1995) mediante la siguiente expresión: 


$$
y=X b+Z_{u} u+Z_{p} p+e
$$

Donde:
Y:
Es el vector de las observaciones para altura o diámetro en cada uno de los sitios
B:
Es el vector de parámetros para los efectos fijos, para este
U: análisis univariado se considera la procedencia y bloque Es el vector de valores genéticos para cada una de las
P: $\quad$ Es el vector de efectos aleatorios de parcela variables dentro de cada sitio, $\mathbf{u} \sim\left(0, \sigma^{2} \mathbf{G}\right)$
(bloque*familia)
e: $\mathbf{Z}_{\mathrm{u}}$ y $\mathbf{Z}_{\mathrm{p}}$ :
Es el vector de residuos aleatorios, $\mathbf{e} \sim\left(0, \sigma^{2} \mathbf{R}\right)$,
Representan respectivamente las matrices de diseño para los efectos fijos, valores genéticos y efectos de parcela.

ASREML (Gilmour et al., 1995) permite que tanto $\mathbf{G}$ y $\mathbf{R}$ se definan como la suma directa de los productos directos de las matrices de covarianza parametrizadas.

El análisis de datos se llevó a cabo para cada variable en cada sitio (es decir un subconjunto del modelo multisitio, incluyendo bloque, procedencia, valor genético del árbol, el efecto parcela y error). Esto permite obtener unos valores que, aunque pueden ser considerados iniciales para los componentes de varianza, permiten alcanzar la mejor predicción lineal insesgada (BLUP) de los valores genéticos usando las ecuaciones de modelos lineales mixtos de Henderson (1984). Una explicación más detallada de los modelos mixtos en Ipinza (1997).

Los componentes de varianza fueron estimados usando Máxima Verosimilitud Restringida (REML) Patterson y Thompson (1971). Para esto se utilizó el programa ASREML (Gilmour et al., 1995, Gilmour et al., 1997), que mediante el uso de un algoritmo iterativo de información promedio y técnicas de sparse matrices reduce en forma considerable los tiempos de análisis y requerimientos computacionales.

Además de los componentes de varianza, se estimó las heredabilidades $\left(h^{2}\right)$, con su correspondiente error estándar (E.E).

$$
\begin{gathered}
h^{2}=\frac{\sigma_{a}^{2}}{\sigma_{a}^{2}+\sigma_{p}^{2}+\sigma_{e}^{2}} \\
E . E=\sqrt{\operatorname{Var}\left(\frac{\sigma_{a}^{2}}{\sigma^{2} f}\right)}=\sqrt{\left(\frac{\sigma_{a}^{2}}{\sigma_{f}^{2}}\right)^{2} \times\left(\frac{\operatorname{Var}\left(\sigma_{a}^{2}\right)}{\sigma_{f}^{4}}+\frac{\operatorname{Var}\left(\sigma_{f}^{2}\right)}{\sigma_{f}^{4}}-\frac{2 \operatorname{Cov}\left(\sigma_{a}^{2}, \sigma_{f}^{2}\right)}{\left(\sigma_{a}^{2} \times \sigma_{f}^{2}\right)}\right)}
\end{gathered}
$$

Los errores estándares de los parámetros genéticos fueron obtenidos directamente con ASREML, basado en los términos de la inversa de la matriz de coeficientes de las ecuaciones de modelos lineales (Gilmour et al., 1997).

\section{Coeficiente de Parentesco (r)}

La biología reproductiva y características ecológicas del género Eucalyptus permiten la existencia de autopolinización y de muy pocos padres contribuyendo con polen. Esto causa relaciones de parentesco más complejas que medios hermanos y conduce a una sobreestimación de las heredabilidades (Squillace 1974, Askew y El-Kassaby, 1994). 
Para enfrentar este problema estos autores han propuesto corregir el coeficiente de parentesco $(r)$ utilizando información de la proporción de autopolinización y del número y características de los progenitores contribuyendo con polen. La corrección depende fuertemente del nivel de autopolinización supuesto.

Un enfoque alternativo fue propuesto por Jarvis et al. (1995) y Borralho y Potts (1996), incluyendo un efecto fijo adicional en el modelo, considerando el tipo de rodal en que fue colectada la semilla establecida en los ensayos. El uso de este método produjo reducciones no significativas de heredabilidad de entre $4 \%$ y $13 \%$ (Borralho y Potts 1996).

Ipinza et al. (1994) utilizan para E. globulus un coeficiente de parentesco (r) de 0,35 para progenies originadas de polinización abierta. Este valor está basado en el supuesto que el $20 \%$ de la progenie de este tipo de ensayo fue originado por autofecundación.

Este coeficiente puede ser leído directamente de la tabla de Squillace (1974) bajo la suposición que hay $20 \%$ de autofecundación y en la polinización participan 30 donantes de polen no emparentados. También a base de este criterio se estimaron las heredabilidades para esta especie obtenidas por Prado y Alvear (1994).

No obstante Potts et al. (1995) recomiendan usar un coeficiente de parentesco de 0,4. Este coeficiente asume una tasa de exogamia del $30 \%$ que es considerada como un promedio para eucalipto, y por esto es solo una aproximación.

Los mencionados autores establecen que el uso de este valor de coeficiente de parentesco es para estandarizar los informes de heredabilidad para ensayos de progenie de polinización abierta de eucalipto.

\section{RESULTADOS}

\section{Supervivencia por Ensayo y Edad}

Para cada uno de los ensayos se muestra en el Cuadro № 4 el número de árboles totales, el número de árboles efectivos y la supervivencia expresada en porcentaje.

En el Cuadro № 4 se aprecia una supervivencia inicial muy alta, la que al transcurrir el tiempo se mantiene a excepción de San Agustín (Región del Maule), donde a partir del mes 71 de vida decae fuertemente, y Los Copihues (Región de Los Ríos), donde a partir del mes 47 se experimenta un aumento en la mortalidad debido a la acción de nevazones.

En el caso de San Agustín los valores de mortalidad no son interpretables directamente, pues se manifestó un severo ataque de Phoracantha semipunctata (Coleóptera, Cerambicidae) y el ensayo fue sometido a un raleo tendiente a eliminar a la mitad de los individuos representados, dejando prevalecer en cada parcela a 2 de los 4 árboles que la conformaban.

El raleo utilizado es un tratamiento silvicultural, por lo bajo es decir se extrajo los individuos débiles o muertos por el insecto. El insecto normalmente ataca a los individuos que presentan un estrés frente a sequía.

Desde el punto de vista genético, el raleo altero la estructura normal de las familias, la sesgo hacia la derecha de la distribución de diámetro o altura. 


\section{SUPERVIVENCIA POR ENSAYO Y EDAD}

\begin{tabular}{|c|c|c|c|c|}
\hline Ensayo & Mes & $\begin{array}{c}\text { ÁrbolesTotales } \\
\left(\mathbf{N}^{\circ}\right)\end{array}$ & $\begin{array}{c}\text { Árboles Efectivos } \\
\left(\mathbf{N}^{\circ}\right)\end{array}$ & $\begin{array}{c}\text { Supervivencia } \\
(\%)\end{array}$ \\
\hline
\end{tabular}

\begin{tabular}{|r|c|r|r|r|}
\hline \multirow{5}{*}{ San Agustín } & 4 & 8.720 & 8.713 & 99,9 \\
\cline { 2 - 5 } & 8 & 8.720 & 8.279 & 94,9 \\
\cline { 2 - 5 } & 21 & 8.720 & 7.922 & 90,8 \\
\cline { 2 - 5 } & 46 & 8.720 & 7.448 & 85,4 \\
\cline { 2 - 5 } (raleo) & 59 & 8.720 & 7.377 & 84,6 \\
\cline { 2 - 5 } & 71 & 8.720 & 3.811 & 43,7 \\
\cline { 2 - 5 } & 83 & 8.720 & 3.382 & 38,8 \\
\hline
\end{tabular}

\begin{tabular}{|c|c|r|r|r|}
\hline & 2 & 8.680 & 8.589 & 98,9 \\
\cline { 2 - 5 } & 6 & 8.680 & 8.343 & 96,1 \\
\cline { 2 - 5 } & 20 & 8.680 & 8.000 & 92,2 \\
\cline { 2 - 5 } & 47 & 8.680 & 6.739 & 77,6 \\
\cline { 2 - 5 } & 67 & 8.680 & 6.646 & 76,6 \\
\hline
\end{tabular}

\begin{tabular}{|l|c|r|r|r|}
\hline & 2 & 7.740 & 7.735 & 99,9 \\
\cline { 2 - 5 } Los Hermanos & 8 & 7.740 & 7.627 & 98,5 \\
\cline { 2 - 5 } & 19 & 7.740 & 7.492 & 96,8 \\
\cline { 2 - 5 } & 44 & 7.740 & 7.311 & 94,5 \\
\cline { 2 - 5 } & 67 & 7.740 & 7.280 & 94,0 \\
\hline
\end{tabular}

\begin{tabular}{|l|r|r|r|r|}
\hline \multirow{4}{*}{ Tanumé } & 6 & 120 & 120 & 100,0 \\
\cline { 2 - 5 } & 9 & 120 & 120 & 100,0 \\
\cline { 2 - 5 } & 21 & 120 & 118 & 98,3 \\
\cline { 2 - 5 } & 45 & 120 & 117 & 97,5 \\
\hline
\end{tabular}

\section{Estadísticos Básicos por Rasgos, Ensayos y Edad}

En los Cuadros № 5 y № 6 se indica los valores mínimos, medios y máximos alcanzados en cada medición para los rasgos altura y diámetro, en cada uno de los ensayos considerados.

La variable altura promedio a los 67 meses exhibe el mayor valor en Los Hermanos (Región del Bio Bio), luego le siguen San Agustín (Región del Maule) y Los Copihues (Región de Los Ríos). También se encuentra la máxima altura individual en el ensayo Los Hermanos. Los valores que muestra Tanumé (Región de O'Higgins) a los 45 meses, aun cuando son menores, resultan interesantes dado que es el ensayo ubicado más al $\mathrm{N}$, zona con las menores precipitaciones medias anuales de los cuatro ensayos.

En el Cuadro № 6, se aprecia que el ensayo de mayor diámetro corresponde también a Los Hermanos a los 67 meses de edad. El ensayo Tanumé muestra para el diámetro las mismas tendencias observadas para la variable altura. Se debe recordar que la zona coloreada del cuadro indica el DAP. 
Cuadro № 5

VALOR MÍNIMO, MEDIA Y VALOR MÁXIMO PARA EL RASGO ALTURA

\begin{tabular}{|l|c|c|c|c|}
\hline \multirow{2}{*}{ Ensayo } & \multirow{2}{*}{ Mes } & Mínimo & Media & Máximo \\
\cline { 3 - 5 } & & \multicolumn{3}{|c|}{$(\mathrm{m})$} \\
\hline
\end{tabular}

\begin{tabular}{|r|r|r|r|r|}
\hline \multirow{4}{*}{ San Agustín } & 4 & 0,100 & 0,411 & 0,900 \\
\cline { 2 - 5 } & 8 & 0,200 & 0,741 & 2,000 \\
\cline { 2 - 5 } & 21 & 0,200 & 2,387 & 5,700 \\
\cline { 2 - 5 } & 46 & 0,300 & 5,082 & 10,500 \\
\cline { 2 - 5 } (raleo) & 59 & 0,900 & 7,153 & 15,000 \\
\cline { 2 - 5 } & 71 & 1,500 & 9,683 & 15,500 \\
\cline { 2 - 5 } & 83 & 2,400 & 12,080 & 18,500 \\
\hline
\end{tabular}

\begin{tabular}{|c|c|r|r|r|}
\hline & 2 & 0,100 & 0,273 & 0,900 \\
\cline { 2 - 5 } Los Copihues & 6 & 0,100 & 0,842 & 1,500 \\
\cline { 2 - 5 } & 20 & 0,300 & 1,651 & 3,800 \\
\cline { 2 - 5 } & 47 & 0,300 & 4,811 & 11,000 \\
\cline { 2 - 5 } & 67 & 0,600 & 9,015 & 15,700 \\
\hline
\end{tabular}

\begin{tabular}{|c|c|r|r|r|}
\hline \multirow{3}{*}{ Los Hermanos } & 2 & 0,100 & 0,275 & 0,700 \\
\cline { 2 - 5 } & 8 & 0,100 & 0,790 & 1,900 \\
\cline { 2 - 5 } & 19 & 0,300 & 2,251 & 5,000 \\
\cline { 2 - 5 } & 44 & 0,300 & 7,724 & 12,500 \\
\cline { 2 - 5 } & 67 & 0,700 & 12,888 & 20,500 \\
\hline
\end{tabular}

\begin{tabular}{|l|c|r|r|r|}
\hline \multirow{4}{*}{ Tanumé } & 6 & 0,400 & 0,869 & 1,200 \\
\cline { 2 - 5 } & 9 & 0,600 & 1,158 & 2,000 \\
\cline { 2 - 5 } & 21 & 1,200 & 2,583 & 4,300 \\
\cline { 2 - 5 } & 45 & 3,400 & 7,561 & 12,000 \\
\hline
\end{tabular}


Cuadro № 6

VALOR MÍNIMO, MEDIA Y VALOR MÁXIMO PARA EL RASGO DIÁMETRO

\begin{tabular}{|l|c|c|c|c|}
\hline \multirow{2}{*}{ Ensayo } & \multirow{2}{*}{ Mes } & Mínimo & Media & Máximo \\
\cline { 3 - 4 } & & \multicolumn{3}{|c|}{$(\mathrm{cm})$} \\
\hline
\end{tabular}

\begin{tabular}{|r|c|r|r|r|}
\hline & 4 & - & - & - \\
\cline { 2 - 5 } San Agustín & 8 & 0,200 & 1,196 & 3,200 \\
\cline { 2 - 5 } & 21 & 0,300 & 3,650 & 9,400 \\
\cline { 2 - 5 } & 46 & 0,300 & 4,711 & 13,000 \\
\cline { 2 - 5 } (raleo) & 59 & 0,400 & 6,185 & 14,600 \\
\cline { 2 - 5 } & 71 & 0,500 & 8,347 & 18,000 \\
\cline { 2 - 5 } & 83 & 2,100 & 11,272 & 25,000 \\
\hline
\end{tabular}

\begin{tabular}{|c|c|r|r|r|}
\hline & 2 & - & - & - \\
\cline { 2 - 5 } & 6 & 0,100 & 0,807 & 1,900 \\
\cline { 2 - 5 } & 20 & 0,300 & 2,121 & 5,700 \\
\cline { 2 - 5 } & 47 & 0,200 & 4,345 & 11,600 \\
\cline { 2 - 5 } & 67 & 0,300 & 8,175 & 19,900 \\
\hline
\end{tabular}

\begin{tabular}{|c|c|r|r|r|}
\hline & 2 & - & - & - \\
\cline { 2 - 5 } Los Hermanos & 8 & 0,200 & 1,288 & 3,200 \\
\cline { 2 - 5 } & 19 & 0,200 & 3,228 & 7,600 \\
\cline { 2 - 5 } & 44 & 0,300 & 6,828 & 13,000 \\
\cline { 2 - 5 } & 67 & 0,300 & 10,772 & 21,000 \\
\hline
\end{tabular}

\begin{tabular}{|l|c|r|r|r|}
\hline \multirow{4}{*}{ Tanumé } & 6 & 0,300 & 0,720 & 1,400 \\
\cline { 2 - 5 } & 9 & 0,600 & 1,395 & 2,700 \\
\cline { 2 - 5 } & 21 & 0,500 & 3,988 & 6,900 \\
\cline { 2 - 5 } & 45 & 2,000 & 6,354 & 13,400 \\
\hline
\end{tabular}

\section{Componentes de Varianza por Rasgos, Ensayos y Edad}

En los Cuadros № 7 y $\mathrm{N}^{\circ} 8$ se indica las estimaciones de los componentes de varianza del efecto árbol $\left(\sigma^{2}\right.$ arbol), del efecto parcela $\left(\sigma^{2}\right.$ parcela), que corresponde a la interacción $\sigma^{2}{ }_{\text {bloque familia }}$ y al error $\left(\sigma^{2}\right.$ error $)$ con sus respectivos errores estándares para los rasgos altura y diámetro en cada una de las edades consideradas.

En el Cuadro № , para la variable altura , se observa que el efecto árbol aumenta en relación directa a la edad, con excepción del ensayo San Agustín (Región del Bio Bio) que muestra a la edad de 71 meses una leve disminución, que coincide con la aplicación del raleo fitosanitario que fue necesario aplicar.

Una proporcionalidad directa también se observa en el efecto parcela y el error para el rasgo altura. No obstante, la componente del error es normalmente más grande. A excepción del ensayo San Agustín que exhibe a los 71 y 83 meses valores similares en el efecto parcela y en el efecto error.

En el ensayo Los Hermanos el efecto árbol es mayor que el efecto parcela, similar tendencia se observa en el ensayo Tanumé. En cambio, en los ensayos San Agustín y Los Copihues el efecto parcela es ligeramente mayor que el efecto árbol. 


\section{Cuadro № 7}

ESTIMACIÓN DE COMPONENTES DE VARIANZA Y ERRORES ESTÁNDARES PARA EL RASGO ALTURA

\begin{tabular}{|l|l|l|l|l|l|l|l|}
\hline \multirow{2}{*}{ Ensayo } & \multirow{2}{*}{ Mes } & $\sigma^{2}{ }_{\text {arbol }}$ & E.E & $\sigma_{\text {parcela }}{ }^{2}$ & E.E & $\sigma^{2}{ }_{\text {error }}$ & E.E \\
\cline { 3 - 8 } & \multicolumn{6}{|c|}{$(\mathrm{m})$} \\
\hline
\end{tabular}

\begin{tabular}{|r|c|r|r|r|r|r|r|}
\hline & 4 & 0,0044 & 0,0005 & 0,0004 & 0,0001 & 0,00207 & 0,000408 \\
\cline { 2 - 8 } San Agustín & 8 & 0,0081 & 0,0014 & 0,0055 & 0,0004 & 0,01947 & 0,001124 \\
\cline { 2 - 8 } & 21 & 0,0707 & 0,0150 & 0,1015 & 0,0058 & 0,21150 & 0,012296 \\
\cline { 2 - 8 } & 46 & 0,2250 & 0,0717 & 0,7086 & 0,0362 & 1,13569 & 0,059585 \\
\cline { 2 - 8 } (raleo) & 59 & 0,5695 & 0,1427 & 1,2237 & 0,0625 & 1,77520 & 0,115423 \\
\cline { 2 - 8 } & 71 & 0,4764 & 0,1777 & 1,5950 & 0,0929 & 1,48845 & 0,146357 \\
\cline { 2 - 8 } & 83 & 0,7718 & 0,2590 & 1,9082 & 0,1290 & 1,99948 & 0,215694 \\
\hline
\end{tabular}

\begin{tabular}{|l|c|r|r|r|r|r|r|}
\hline \multirow{4}{*}{ Los } & 2 & 0,0006 & - & 0,0001 & - & 0,00000 & - \\
\cline { 2 - 8 } & 6 & 0,0056 & 0,0010 & 0,0044 & 0,0003 & 0,01498 & 0,000822 \\
\cline { 2 - 8 } & 20 & 0,0364 & 0,0066 & 0,0312 & 0,0021 & 0,09101 & 0,005398 \\
\cline { 2 - 8 } & 47 & 0,4401 & 0,1076 & 0,8415 & 0,0454 & 1,23034 & 0,087011 \\
\cline { 2 - 8 } & 67 & 1,2963 & 0,2542 & 1,3988 & 0,0879 & 2,60992 & 0,204700 \\
\hline
\end{tabular}

\begin{tabular}{|l|c|r|r|r|r|r|r|}
\hline & 2 & 0,0009 & 0,0444 & 0,0003 & 0,0154 & 0,00000 & - \\
\cline { 2 - 8 } Los & 8 & 0,0126 & 0,0020 & 0,0074 & 0,0005 & 0,01310 & 0,001558 \\
\cline { 2 - 8 } Hermanos & 19 & 0,1023 & 0,0191 & 0,1077 & 0,0059 & 0,15948 & 0,015046 \\
\cline { 2 - 8 } & 44 & 0,8174 & 0,1417 & 0,5314 & 0,0397 & 1,58580 & 0,114333 \\
\cline { 2 - 8 } & 67 & 3,4101 & 0,4858 & 0,6692 & 0,0901 & 4,13581 & 0,386886 \\
\hline
\end{tabular}

\begin{tabular}{|l|c|r|r|r|r|r|r|}
\hline \multirow{4}{*}{ Tanumé } & 6 & 0,0012 & 0,0002 & 0,0123 & 0,0052 & 0,01210 & 0,001803 \\
\cline { 2 - 8 } & 9 & 0,0039 & 0,0006 & 0,0095 & 0,0069 & 0,03910 & 0,005827 \\
\cline { 2 - 8 } & 21 & 0,0240 & 0,0036 & 0,0021 & 0,0260 & 0,24007 & 0,036155 \\
\cline { 2 - 8 } & 45 & 0,2115 & 0,0321 & 0,1822 & 0,2719 & 2,11537 & 0,320511 \\
\hline
\end{tabular}

$\sigma_{\text {parcela }}^{2}=\sigma^{2}$ bloque ${ }^{*}$ familia

Para el diámetro, en el Cuadro № 8 se observa que las tendencias de la altura se mantienen. El efecto parcela sigue siendo mayor en el ensayo San Agustín. relación se invierte.

Similar tendencia se observa en Los Copihues, salvo en la última medición donde la

El ensayo San Agustín y Los Copihues son ensayos localizados en sitios muy heterogéneos.

En el ensayo Los Hermanos el efecto árbol es claramente mayor, en cambio en el ensayo Tanumé ambos efectos presentan niveles similares. 
Cuadro № 8

ESTIMACIÓN DE COMPONENTES DE VARIANZA Y ERRORES ESTÁNDARES PARA EL RASGO DIÁMETRO

\begin{tabular}{|l|l|l|l|l|l|l|l|}
\hline \multirow{2}{*}{ Ensayo } & \multirow{2}{*}{ Mes } & $\sigma_{\text {arbol }}^{2}$ & E.E & $\sigma_{\text {parcela }}{ }^{1}$ & E.E & $\sigma_{\text {error }}^{2}$ & E.E \\
\cline { 3 - 6 } & & \multicolumn{7}{|c|}{$(\mathbf{c m})$} &
\end{tabular}

\begin{tabular}{|r|c|r|r|r|r|r|r|}
\hline & 4 & - & - & - & - & - & - \\
\cline { 2 - 8 } San Agustín & 8 & 0,0154 & 0,0035 & 0,0196 & 0,0014326 & $7,65 \mathrm{E}-02$ & 0,0030469 \\
\cline { 2 - 8 } & 21 & 0,1949 & 0,0414 & 0,2454 & 0,0161126 & 0,71512 & 0,0349520 \\
\cline { 2 - 8 } & 46 & 0,2900 & 0,0884 & 0,6998 & 0,0440386 & 1,87928 & 0,0779461 \\
\cline { 2 - 8 } (raleo) & 59 & 0,7708 & 0,1630 & 0,9777 & 0,0625900 & 2,39704 & 0,1356559 \\
\cline { 2 - 8 } & 71 & 0,9439 & 0,2818 & 1,9628 & 0,1363975 & 2,56667 & 0,2367777 \\
\cline { 2 - 8 } & 83 & 1,0355 & 0,3659 & 2,5029 & 0,1920860 & 3,48908 & 0,3148989 \\
\hline
\end{tabular}

\begin{tabular}{|c|c|r|r|r|r|r|r|}
\hline & 2 & - & - & - & - & - & - \\
\cline { 2 - 8 } & 6 & 0,0057 & 0,0011 & 0,0053 & 0,000415 & $2,27 \mathrm{E}-02$ & 0,0009889 \\
\cline { 2 - 8 } & 20 & 0,0730 & 0,0166 & 0,0979 & 0,0067586 & 0,325803 & 0,0142708 \\
\cline { 2 - 8 } & 47 & 0,6923 & 0,1584 & 1,1138 & 0,063899 & 1,86349 & 0,128783 \\
\cline { 2 - 8 } & 67 & 2,6701 & 0,4636 & 1,7997 & 0,1370663 & 4,65017 & 0,3744098 \\
\hline
\end{tabular}

\begin{tabular}{|c|c|r|r|r|r|r|r|}
\hline & 2 & - & - & - & - & - & - \\
\cline { 2 - 8 } & 8 & 0,0178 & 0,0051 & 0,0422 & 0,0023721 & $8,83 \mathrm{E}-02$ & 0,0042947 \\
\cline { 2 - 8 } & 19 & 0,1809 & 0,0380 & 0,2351 & 0,0137554 & 0,477626 & 0,0308146 \\
\cline { 2 - 8 } & 44 & 1,5099 & 0,2367 & 0,6696 & 0,0559363 & 2,23973 & 0,1891664 \\
\cline { 2 - 8 } & 67 & 5,3782 & 0,7367 & 0,6102 & 0,1220346 & 6,0005 & 0,5859863 \\
\hline
\end{tabular}

\begin{tabular}{|l|c|r|r|r|r|r|r|}
\hline \multirow{4}{*}{ Tanumé } & 6 & 0,0026 & 0,0004 & 0,0093 & 0,0055536 & $2,62 \mathrm{E}-02$ & 0,0039046 \\
\cline { 2 - 8 } & 9 & 0,0099 & 0,0015 & 0,0192 & 0,0157377 & $9,90 \mathrm{E}-02$ & 0,0147541 \\
\cline { 2 - 8 } & 21 & 0,0896 & 0,0135 & 0,0737 & 0,1116667 & 0,89579 & 0,1351116 \\
\cline { 2 - 8 } & 45 & 0,3144 & 0,0476 & 0,1489 & 0,3631659 & 3,14433 & 0,4764136 \\
\hline
\end{tabular}

${ }^{1} \sigma_{\text {parcela }}^{2}=\sigma^{2}$ bloque ${ }^{*}$ familia

\section{Nivel de Importancia de los Componentes de Varianza por Rasgos, Ensayos y Edad}

En los Cuadros $\mathrm{N}^{\circ} 9$ y $\mathrm{N}^{\circ} 10$ se determinan los niveles de explicación de los componentes de varianza en relación a la varianza fenotípica para el rasgo altura y diámetro, respectivamente.

En el Cuadro $N^{\circ} 9$ se muestra que para la altura el efecto aditivo del árbol tiende a disminuir su importancia a medida que progresa la edad, salvo en sus mediciones finales donde ocurre un incremento. Una excepción a esta tendencia se muestra en Tanumé con un ligero incremento desde el inicio. El efecto parcela se incrementa desde el inicio en San Agustín y Los Copihues y luego disminuye ligeramente. Los Hermanos, presenta una conducta más errática aunque al final alcanza niveles muy bajos. El ensayo Tanumé también muestra una fuerte disminución.

La conducta del efecto error es más compleja, ya que siempre presenta un mayor nivel de explicación en las mediciones finales de altura en cada uno de los ensayos, pero es dentro del ensayo Tanumé donde se muestran los mayores valores. 


\section{Cuadro $\mathrm{N}^{\circ} 9$}

NIVELES DE EXPLICACIÓN DE LOS COMPONENTES DE VARIANZA ${ }^{1}$ EN RELACIÓN A LA VARIACIÓN FENOTÍPICA ${ }^{2}$ PARA EL RASGO ALTURA

\begin{tabular}{|l|l|l|l|l|}
\hline Ensayo & Mes & $\left(\sigma_{\text {arbol }}^{2} / \sigma_{\text {fenotipica }}^{2}\right)^{*} 100$ & $\left(\sigma_{\text {parcela }}^{2} / \sigma_{\text {fenotipica }}^{2}\right)^{*} 100$ & $\left(\sigma_{\text {error }}^{2} / \sigma_{\text {fenotipica }}^{2}\right)^{*} 100$ \\
\hline
\end{tabular}

\begin{tabular}{|r|c|r|r|r|}
\hline \multirow{4}{*}{ San Agustín } & 4 & 64,24 & 5,46 & 30,30 \\
\cline { 2 - 5 } & 8 & 24,43 & 16,64 & 59,01 \\
\cline { 2 - 5 } & 21 & 18,43 & 26,45 & 55,13 \\
\cline { 2 - 5 } & 46 & 10,88 & 34,25 & 54,89 \\
\cline { 2 - 5 } (raleo) & 59 & 15,96 & 34,30 & 49,75 \\
\cline { 2 - 5 } & 71 & 13,38 & 44,80 & 41,81 \\
\cline { 2 - 5 } & 83 & 16,49 & 40,77 & 42,72 \\
\hline
\end{tabular}

\begin{tabular}{|l|c|r|r|r|}
\hline \multirow{4}{*}{ Los Copihues } & 2 & 85,94 & 13,99 & 0,01 \\
\cline { 2 - 5 } & 6 & 22,42 & 17,53 & 60,14 \\
\cline { 2 - 5 } & 20 & 22,94 & 19,68 & 57,42 \\
\cline { 2 - 5 } & 47 & 17,52 & 33,50 & 48,98 \\
\cline { 2 - 5 } & 67 & 24,44 & 26,37 & 49,20 \\
\hline
\end{tabular}

\begin{tabular}{|l|c|r|r|r|}
\hline & 2 & 74,00 & 25,58 & 0,00 \\
\cline { 2 - 5 } Los Hermanos & 8 & 38,07 & 22,33 & 39,58 \\
\cline { 2 - 5 } & 19 & 27,69 & 29,15 & 43,15 \\
\cline { 2 - 5 } & 44 & 27,85 & 18,10 & 54,03 \\
\cline { 2 - 5 } & 67 & 41,51 & 8,15 & 50,34 \\
\hline
\end{tabular}

\begin{tabular}{|l|c|r|r|r|}
\hline \multirow{4}{*}{ Tanumé } & 6 & 4,75 & 48,24 & 47,45 \\
\cline { 2 - 5 } & 9 & 7,46 & 18,05 & 74,62 \\
\cline { 2 - 5 } & 21 & 9,02 & 0,78 & 90,22 \\
\cline { 2 - 5 } & 45 & 8,43 & 7,26 & 84,31 \\
\hline
\end{tabular}

${ }^{1}$ Varianza $\left(\sigma_{\text {arbol }}^{2}, \sigma_{\text {parcela }}^{2}=\sigma_{\text {bloque tamilia }}^{2} \sigma^{2}{ }_{\text {error }}\right)$

${ }^{2}$ Variación fenotípica $\left(\sigma_{\text {fenotipica }}^{2}=\sigma_{\text {arbol }}^{2}+\sigma_{\text {parcela }}^{2}+\sigma_{\text {error }}^{2}\right)$

En el Cuadro $\mathrm{N}^{\circ} 10$, se aprecia la importancia del efecto árbol para la variable diámetro, la que es bastante fluctuante en el ensayo San Agustín, algo errática en los Copihues y en cambio en Los Hermanos y Tanumé aumenta sistemáticamente.

El efecto parcela tiende a aumentar en San Agustín, aumenta a cierta edad en Los Copihues y disminuyen sistemáticamente en Los Hermanos y Tanumé.

La importancia del efecto error a medida que progresa la edad en general tiende a la disminución a excepción del ensayo Tanumé en el que tiende a aumentar. 


\section{Cuadro $\mathrm{N}^{\circ} 10$ \\ NIVELES DE EXPLICACIÓN DE LOS COMPONENTES DE VARIANZA ${ }^{1}$ EN RELACIÓN A LA VARIACIÓN FENOTÍPICA ${ }^{2}$ PARA EL RASGO DIÁMETRO}

\begin{tabular}{|l|l|l|l|l|}
\hline Ensayo & Mes & $\left(\sigma_{\text {arbol }}^{2} / \sigma_{\text {fenotipica }}^{2}\right)^{\star} 100$ & $\left(\sigma_{\text {parcela }}^{2} / \sigma_{\text {fenotipica }}^{2}\right)^{*} 100$ & $\left(\sigma_{\text {error }}^{2} / \sigma_{\text {fenotipica }}^{2}\right)^{\star} 100$ \\
\hline
\end{tabular}

\begin{tabular}{|r|c|r|r|r|}
\hline \multirow{5}{*}{ San Agustín } & 4 & - & - & - \\
\cline { 2 - 5 } & 8 & 13,81 & 17,54 & 68,64 \\
\cline { 2 - 5 } & 21 & 16,88 & 21,25 & 61,91 \\
\cline { 2 - 5 } & 46 & 10,11 & 24,39 & 65,50 \\
\cline { 2 - 5 } (raleo) & 59 & 18,59 & 23,58 & 57,82 \\
\cline { 2 - 5 } & 71 & 17,25 & 35,86 & 46,90 \\
\cline { 2 - 5 } & 83 & 14,74 & 35,62 & 49,65 \\
\hline
\end{tabular}

\begin{tabular}{|l|c|r|r|r|}
\hline & 2 & - & - & - \\
\cline { 2 - 5 } & 6 & 16,97 & 15,65 & 67,32 \\
\cline { 2 - 5 } & 20 & 14,71 & 19,70 & 65,59 \\
\cline { 2 - 5 } & 47 & 18,86 & 30,35 & 50,78 \\
\cline { 2 - 5 } & 67 & 29,28 & 19,73 & 50,99 \\
\hline
\end{tabular}

\begin{tabular}{|l|c|r|r|r|}
\hline & 2 & - & - & - \\
\cline { 2 - 5 } & 8 & 12,01 & 28,48 & 59,58 \\
\cline { 2 - 5 } Los Hermanos & 19 & 20,25 & 26,30 & 53,44 \\
\cline { 2 - 5 } & 44 & 34,17 & 15,15 & 50,68 \\
\cline { 2 - 5 } & 67 & 44,86 & 5,09 & 50,05 \\
\hline
\end{tabular}

\begin{tabular}{|l|c|r|r|r|}
\hline \multirow{4}{*}{ Tanumé } & 6 & 6,86 & 24,42 & 68,59 \\
\cline { 2 - 6 } & 9 & 7,73 & 14,99 & 77,28 \\
\cline { 2 - 5 } & 21 & 8,46 & 6,96 & 84,59 \\
\cline { 2 - 5 } & 45 & 8,71 & 4,13 & 87,15 \\
\hline
\end{tabular}

${ }^{1}$ Varianza $\left(\sigma_{\text {arbol }}^{2}, \sigma_{\text {parcela }}^{2}=\sigma_{\text {bloque tamilia }}^{2} \sigma_{\text {error }}^{2}\right)$

${ }^{2}$ Variación fenotípica $\left(\sigma^{2}\right.$ fenotipica $\left.=\sigma_{\text {arbol }}^{2}+\sigma_{\text {parcela }}^{2}+\sigma_{\text {error }}^{2}\right)$

\section{Heredabilidad por Rasgos, Ensayos y Edad}

En los Cuadros $N^{\circ} 11$ y $N^{\circ} 12$ se muestra la variación fenotípica $\left(\sigma_{\text {fenotipica }}^{2}\right.$, la varianza aditiva $\left(\boldsymbol{\sigma}_{\text {aditiva }}^{2}\right)$, la heredabilidad individual $\left(\mathbf{h}_{\mathrm{i}}^{2}\right)$ y su respectivos errores estándares para la variable altura y diámetro, respectivamente.

En el Cuadro $N^{\circ} 11$ se aprecia que la heredabilidad de la altura tiende a disminuir con la edad. No obstante, la última edad exhibe un incremento, que es muy importante en el ensayo Los Hermanos, alcanzando una heredabilidad de 0,26 $(E=0,03)$.

Si se excluye Tanumé, las heredabilidad en altura fluctúa entre 0,10 a 0,25 . Para el diámetro los valores son algo superiores y fluctúan entre 0,11 a 0,28. 
Cuadro $\mathrm{N}^{\circ} 11$

ESTIMACIÓN DE LA VARIANZA FENOTÍPICA TOTAL ${ }^{1}$, VARIANZA ADITIVA ${ }^{2}$, HEREDABILIDAD INDIVIDUAL ${ }^{3}$ Y SU RESPECTIVO ERROR ESTÁNDAR PARA EL RASGO ALTURA

\begin{tabular}{|l|l|l|l|l|l|}
\hline Ensayo & Mes & $\sigma_{\text {fenotipica }}^{2}$ & $\sigma_{\text {aditiva }}^{2}$ & $\mathbf{h}^{2}{ }_{\mathrm{i}}$ & E.E \\
\hline
\end{tabular}

\begin{tabular}{|r|c|r|r|r|r|}
\hline \multirow{4}{*}{ San Agustín } & 4 & 0,0068 & 0,0027 & 0,4015 & 0,0414 \\
\cline { 2 - 6 } & 8 & 0,0330 & 0,0050 & 0,1526 & 0,0246 \\
\cline { 2 - 6 } & 21 & 0,3836 & 0,0442 & 0,1152 & 0,0237 \\
\cline { 2 - 6 } & 46 & 2,0690 & 0,1406 & 0,0680 & 0,0214 \\
\cline { 2 - 6 } (raleo) & 59 & 3,5680 & 0,3559 & 0,0997 & 0,0243 \\
\cline { 2 - 6 } & 71 & 3,5600 & 0,2977 & 0,0836 & 0,0307 \\
\cline { 2 - 6 } & 83 & 4,6800 & 0,4824 & 0,1031 & 0,0339 \\
\hline
\end{tabular}

\begin{tabular}{|c|c|c|c|c|r|}
\hline & 2 & 0,0006 & 0,0003 & 0,5374 & ${ }^{*}$ \\
\cline { 2 - 6 } & 6 & 0,0249 & 0,0035 & 0,1400 & 0,0239 \\
\cline { 2 - 6 } & 20 & 0,1585 & 0,0227 & 0,1433 & 0,0248 \\
\cline { 2 - 6 } & 47 & 2,5120 & 0,2750 & 0,1095 & 0,0261 \\
\cline { 2 - 6 } & 67 & 5,3050 & 0,8102 & 0,1527 & 0,0286 \\
\hline
\end{tabular}

\begin{tabular}{|c|c|r|r|r|r|}
\hline & 2 & 0,0012 & 0,0006 & 0,4644 & 0,0253 \\
\cline { 2 - 6 } & 8 & 0,0331 & 0,0079 & 0,2386 & 0,0347 \\
\cline { 2 - 6 } Los Hermanos & 19 & 0,3696 & 0,0640 & 0,1731 & 0,0307 \\
\cline { 2 - 6 } & 44 & 2,9350 & 0,5108 & 0,1741 & 0,0285 \\
\cline { 2 - 6 } & 67 & 8,2150 & 2,1310 & 0,2594 & 0,0336 \\
\hline
\end{tabular}

\begin{tabular}{|c|c|c|c|c|r|}
\hline \multirow{4}{*}{ Tanumé } & 6 & 0,0255 & 0,0008 & 0,0295 & 0,0065 \\
\cline { 2 - 6 } & 9 & 0,0524 & 0,0024 & 0,0466 & 0,0065 \\
\cline { 2 - 6 } & 21 & 0,2661 & 0,0150 & 0,0564 & 0,0052 \\
\cline { 2 - 6 } & 45 & 2,5090 & 0,1322 & 0,0527 & 0,0059 \\
\hline
\end{tabular}

\footnotetext{
${ }^{1}$ Varianza fenotípica total $\left(\sigma^{2}\right.$ fenotípica $)$

${ }^{2}$ Varianza aditiva $\left(\sigma^{2}\right.$ aditiva)

${ }^{3}$ Heredabilidad individual $\left(\mathrm{h}^{2}{ }_{\mathrm{i}}\right)$

* Valores muy bajos
} 


\section{ESTIMACIÓN DE LA VARIANZA FENOTÍPICA TOTAL ${ }^{1}$, VARIANZA ADITIVA ${ }^{2}$, HEREDABILIDAD INDIVIDUAL ${ }^{3}$ Y SU RESPECTIVO ERROR ESTÁNDAR PARA EL RASGO DIÁMETRO}

\begin{tabular}{|l|c|c|c|c|c|}
\hline Ensayo & Mes & $\sigma_{\text {fenotipica }}^{2}$ & $\sigma_{\text {aditiva }}^{2}$ & $\mathbf{h}_{\mathbf{i}}^{2}$ & E.E \\
\hline
\end{tabular}

\begin{tabular}{|l|c|r|r|r|r|}
\hline \multirow{4}{*}{ San Agustín } & 4 & - & - & - & - \\
\cline { 2 - 6 } & 8 & 0,1115 & 0,0096 & 0,0863 & 0,0189 \\
\cline { 2 - 6 } & 21 & 1,1550 & 0,1218 & 0,1054 & 0,0217 \\
\cline { 2 - 6 } & 46 & 2,8690 & 0,1813 & 0,0632 & 0,0190 \\
\cline { 2 - 6 } (raleo) & 59 & 4,1460 & 0,4818 & 0,1162 & 0,0238 \\
\cline { 2 - 6 } & 71 & 5,4730 & 0,5899 & 0,1078 & 0,0314 \\
\cline { 2 - 6 } & 83 & 7,0270 & 0,6472 & 0,0921 & 0,0320 \\
\hline
\end{tabular}

\begin{tabular}{|c|c|r|r|r|r|}
\hline & 2 & - & - & - & - \\
\cline { 2 - 6 } & 6 & 0,0337 & 0,0036 & 0,1061 & 0,0207 \\
\cline { 2 - 6 } & 20 & 0,4967 & 0,0457 & 0,0919 & 0,0203 \\
\cline { 2 - 6 } & 47 & 3,6700 & 0,4327 & 0,1179 & 0,0262 \\
\cline { 2 - 6 } & 67 & 9,1200 & 1,6690 & 0,1830 & 0,0300 \\
\hline
\end{tabular}

\begin{tabular}{|c|c|r|r|r|r|}
\hline & 2 & - & - & - & - \\
\cline { 2 - 6 } & 8 & 0,1482 & 0,0111 & 0,0748 & 0,0213 \\
\cline { 2 - 6 } & 19 & 0,8937 & 0,1131 & 0,1266 & 0,0257 \\
\cline { 2 - 6 } & 44 & 4,4190 & 0,9437 & 0,2135 & 0,0311 \\
\cline { 2 - 6 } & 67 & 11,9900 & 3,3610 & 0,2804 & 0,0346 \\
\hline
\end{tabular}

\begin{tabular}{|l|c|r|r|r|r|}
\hline \multirow{4}{*}{ Tanumé } & 6 & 0,0382 & 0,0016 & 0,0429 & 0,0067 \\
\cline { 2 - 6 } & 9 & 0,1281 & 0,0062 & 0,0483 & 0,0063 \\
\cline { 2 - 6 } & 21 & 1,0590 & 0,0560 & 0,0529 & 0,0058 \\
\cline { 2 - 6 } & 45 & 3,6080 & 0,1965 & 0,0545 & 0,0056 \\
\hline
\end{tabular}

${ }^{1}$ Varianza fenotípica total $\left(\sigma^{2}\right.$ fenotipica $)$

${ }^{2}$ Varianza aditiva $\left(\sigma^{2}\right.$ aditiva)

${ }^{3}$ Heredabilidad individual $\left(\mathrm{h}_{\mathrm{i}}^{2}\right)$

\section{DISCUSIÓN Y ANÁLISIS DE RESULTADOS}

Eucalyptus globulus representa la especie de mayor interés para el cultivo de eucaliptos en Chile, dadas sus bondades en la producción de fibra corta que a su vez origina uno de los papeles de mayor calidad. También se destaca por la obtención de otros productos industriales de alto valor agregados, tales como chapas, parquet, aceites esenciales y otros. Además su amplia capacidad de adaptación a distintas situaciones ecológicas, que deben incluir zonas libres de heladas y la ausencia de plagas y enfermedades relevantes le confieren características únicas para su cultivo.

La productividad forestal de la especie está influenciada por varios factores, de los cuales los más relevantes están ligados a la calidad genética del material reproductivo. Es claro que los análisis individuales tienden a sobrestimar los parámetros genéticos, tales como la heredabilidad, no obstante son un buen punto de partida en la caracterización de la estructura genética de las poblaciones bases de la especie en Chile.

La estimación de los parámetros genéticos permite obtener información para conocer la 
naturaleza de la acción génica, envuelta en la herencia de los caracteres, y proporcionar las bases para la definición de los programas de mejora de la población, además de dirigir adecuadamente los esquemas de selección y la evaluación de los progresos esperados a través de los mismos.

La mejor forma de conocer el valor genético de los árboles es a través de ensayos de progenie instalados tanto a partir de semilla de polinización abierta como de cruzamientos controlados. Esto también permite estimar los parámetros genéticos, posibilitando de esta forma la selección de nuevos individuos superiores.

Los parámetros genéticos se obtienen a partir de la estimación de los componentes de varianza del ensayo evaluado. Aunque existen muchos procesos para estimar los componentes de varianza, el de la máxima verosimilitud restringida (REML) es el más apropiado para trabajar con ensayos de progenie. Este método no requiere una demanda especial sobre el tipo de diseño o sobre el balance de los datos.

Uno de los objetivos más comunes de obtener las varianzas genéticas es la estimación de la heredabilidad. Este parámetro se puede definir como la relación entre la varianza genética aditiva y la varianza total, que puede ser atribuida a un efecto medio de los genes. El conocimiento de la heredabilidad es fundamental en los programas de mejora, ya que permite la predicción de las ganancias genéticas, auxiliando en la selección y establecimiento de métodos apropiados de mejora para las especies estudiadas.

En términos generales, en los cuatro ensayos analizados se observa heredabilidades individuales moderadas para diámetro y altura, siendo ligeramente más alta para el DAP $(0,11$ a $0,28)$ que para la altura $(0,10$ a 0,26$)$. En su extremo superior estos valores son similares en magnitud a los señalados por Cornelius (1994) en un compendio de valores de heredabilidad obtenidos por diversos autores. En este documento se señala como el valor medio de heredabilidad individual para altura 0,28 y para el diámetro 0,23 , verificándose una tendencia inversa la observada en este estudio, donde la heredabilidad del diámetro resulta mayor que la de la altura. A este respecto, un estudio similar realizado con ensayos de Eucalyptus nitens (Ipinza et al, 1997) señala una tendencia inversa a esta y coincidente con la señalada por Cornelius (1994).

Las fluctuaciones observadas en los valores de heredabilidad durante el tiempo son consecuencia de los valores adoptados por la varianza fenotípica y la varianza genética aditiva. Estos dos parámetros se presentan crecientes en término absolutos en función de la edad. El primero (varianza fenotípica) es creciente debido a la acumulación de los efectos ambientales sobre las plantas, las cuales en un comienzo son muy homogéneas. El segundo (varianza genética aditiva) es creciente como consecuencia de la expresión progresiva de los genes en la medida que la planta se desarrolla. Como ambas variaciones crecen en distinta proporción, su cuociente, que representa a la heredabilidad, adquiere valores fluctuantes. No obstante, en término relativos, la varianza genética explica para la altura el $24,4 \%$ y $41,5 \%$ de la variación fenotípica en Los Copihues y Los Hermanos, respectivamente, valores muy interesantes al relacionarlos con el nivel de heredabilidad que se alcanza para este rasgo en Los Hermanos $h^{2}=0,26 \pm 0,03$. De la misma forma para el DAP, la varianza genética aditiva explica a la misma edad un $29 \%$ y un $45 \%$ de la variación fenotípica observada para Los Copihues y Los Hermanos, respectivamente, y permitiendo que se alcance una $\mathrm{h}^{2}=0,28 \pm 0,03$ en el ensayo Los Hermanos.

La tendencia general a la disminución de la heredabilidad individual de la altura con el tiempo, obedece a que la varianza genética aditiva crece a una tasa menor que la varianza fenotípica. Por el contrario, en el caso del diámetro los valores registrados para la heredabilidad deben ser analizados con mayor precaución ya que hay dos diámetros el DAC y el DAP. Además, en el caso de San Agustín, la acción del raleo en el mes 71 afecta la tasa de crecimiento en el DAP y como el raleo fue por lo bajo, altera también la estructura dela normalidad de las familias. Este último elemento determina que las estimaciones desde el mes 71 en adelante para propósitos de determinar la estructura genética tienen una validez cuestionable. 
La disminución de la heredabilidad con el aumento de la edad podría finalmente explicarse tanto por la disminución relativa de la cantidad de variación genética como por el aumento de la variación fenotípica. Al respecto, Franklin (1979) lo explica indicando que la disminución es debida a los efectos de la competencia entre las plantas, ya que conforme aumenta la competencia entre ellas la heredabilidad disminuye, aunque ese valor puede crecer nuevamente a medida que los árboles llegan a la madurez.

Los valores de $\mathrm{h}^{2}$ alcanzados en algunos ensayos son adecuados. Evitar los problemas de maleza que tuvieron que soportar en el pasado, unido a las nuevas técnicas de diseños de ensayos, más sensibles a la estimación de estos parámetros genéticos, sugiere que es posible mejorar aún más la expresión de la varianza aditiva y, por tanto, mejorar la heredabilidad de los caracteres de importancias económica como la altura y el DAP. En términos prácticos esto puede conducir a un reposicionamiento de los huertos semilleros de polinización abierta e incluso de la silvicultura familiar en esta especie.

\section{REFERENCIAS}

Askew, G. R. y El- Kassaby, Y. A., 1994. Estimation of relationship coefficients among progeny derived from wind-pollinated orchard seeds. Theoretical and Applied Genetics 88:267-272.

Borralho, N., 1995. The impact of individual tree mixed model methods (BLUP) in tree breeding strategies. En: Eucalyptus Plantation: Improving Fibre Yield and Quality (Eds. B. Potts, N. Borralho, J. Reid, R. Cromer, W. Tibbits y C. Raymond). pp. 141-145. Proc. CRC-IUFRO. Conf., Hobart, 19-24 Feb. CRC for Temperate Hardwood Forestry, Hobart (Australia).

Borralho, N. M. G. y Potts, B. M., 1996. Accounting for native stand characteristics in genetic evaluations of open-pollinated progeny from a Eucalyptus globulus base population. New Forests 11:53-64.

Catterill, P. y Brolin, A., 1997. Improving Eucalyptus Wood, Pulp and Paper Quality by Genetic Selection. IUFROConference on Silviculture and Improvement of Eucalyptus. Salvador, Brazi. 24 - 29 August 1997. Vol. 1. $1-13$.

Cornelius, J., 1994. Heretabilities and aditive genetic coefficients of variation in forest trees. Can. Jour. For. Res. Vol 24 (2): 372-379.

Droppelmann, F. y Delmastro, R. ,1988. Plan de mejoramiento genético de Eucalyptus globulus. Cooperativa de Mejoramiento Genético UACH/CONAF/Empresas Forestales. Universidad Austral de Chile, Facultad de Ciencias Forestales. Valdivia. $22 \mathrm{p}$.

Franklin, E.,1979. Model relating levels of genetic variance to stand development of North American Conifers. Silvae genetica, 28(5/6):207-212.

Gardiner, C. y Crawford, D., 1988. Seed Collection of Eucalyptus globulus ssp. globulus for the Tre Improvement Purposes. Australian Tre Seed Centre. CSIRO. Canberra. Australia.

Gilmour, A. R.; Thompson, R. y Cullis, B. R., 1995. Average information REML, an efficient algorithm for variance parameter estimation in linear mixed models. Biometrics 51:1440-1450.

Gilmour, A. R.; Thompson, R.; Cullis, B. R. y Welham, S.J., 1997. ASREML User's manual. July 24, 1997. $100 \mathrm{p}$.

Griffin, R., 1994. Potential for genetic improvement of Eucalyptus in Chile. En: Actas Simposio Los eucaliptos en el Desarrollo Forestal de Chile. (Barros, S., Prado, J. y Alvear, C. editores). Pucón, Chile 24 al 26 de Noviembre de 1993. INFOR - CORFO. 1 - 25 p.

Henderson, C. R., 1984. Applications of linear models in animal breeding. University of Guelph Press, Guelph.

Infante, P.; Ipinza, R. y Prado, J., 1991. Bases para la mejora genética de las especies del género Eucalyptus en Chile. Ciencia e Investigación Forestal 5(1):96-150. 
INFOR, 1997. Estadísticas Forestales 1996. Boletín estadístico № 50, Septiembre de 1997.

Ipinza, R.; White, T. y Apiolaza, L., 1994. Revisión de la Estrategia de Eucalyptus globulus. Cooperativa de Mejoramiento Genético. UACH/CONAF/Empresas Forestales. Valdivia, Octubre de 1994. 40 p. + anexos.

Ipinza, R., Gutiérrez, B. y Molina, M., 1997. Análisis genético univariado de siete ensayos de progenie y procedencia de Eucalyptus nitens (Deane \& Maiden) Maiden, en Chile. Potencial genético y Silvícola. En IUFRO Conference Modelling Growth of Fast Growing Tree Species. 3-5 September 1997. Valdivia - Chile.

Ipinza, R., 1997. Curso Corto: Mejoramiento Genético Forestal Operativo. - Universidad del Valle, Cali, Colombia. Septiembre 22 al 26 de 1997. 500 p.

Jarvis, S. F.; Borralho, N. M. G. y Potts, B.M. , 1995. Implementation of a multivariate BLUP model for genetic evaluation of Eucalyptus globulus in Australia. En: "Eucalypt plantations: improving fibre yield and quality" (Eds. B.M. Potts, N.M.G. Borralho, J.B. Reid, R.N. Cromer, W.N. Tibbits and C.A. Raymond). pp. 212-216. Proc. CRCIUFRO Conf., Hobart, 19-24 Feb.

Kellison, R., 1997. Production Forestry into the $21^{\text {a }}$ Century: A World View (KEYNOTE). En: $24^{\text {th }}$ Biennial Southern Forest Tree Improvement Conference. Orlando, Florida, USA. June 9-12, 1997. 3-10p.

Patterson, H. D. y Thompson, R. , 1971. Recovery of inter-block information when block sizes are unequal. Biometrika 58:545-554.

Potts, B., Volker, P., Hodge, G., Borralho, N., Hardner, C. y Owen, J. , 1995. Genetic limitations in the exploitation of base populations of Eucalyptus globulus ssp. globulus. En: Eucalyptus Plantation: Improving Fibre Yield and Quality (Eds. B. Potts, N. Borralho, J. Reid, R. Cromer, W. Tibbits y C. Raymond). pp. 217-221. Proc. CRC-IUFRO. Conf., Hobart, 19-24 Feb. CRC for Temperate Hardwood Forestry, Hobart (Australia).

Prado, J., 1988. Selección de procedencias de varias especies del género Eucalyptus para la zona centro - sur de Chile. Actas Simposio Manejo Silvícola del Género Eucalyptus. CORFO - INFOR, Viña del Mar, Chile, Junio 1988. 32 p.

Prado, J. y Alvear, C., 1994. Resultados de ensayos de procedencia y progenies de Eucalyptus globulus en la zona centro - sur de Chile. En: Actas simposio Los eucaliptos en el Desarrollo Forestal de Chile. (Barros, S., Prado, J. y Alvear, C. editores). Pucón, Chile 24 al 26 de Noviembre de 1993. INFOR - CORFO. 61 - 79 p.

Squillace, A. E., 1974. Average genetic correlations among offspring from open-pollinated forest trees. Silvae Genetica 23: 149-156.

Tibbits, W., Boomsma, D. y Jarvis, S., 1997. Distribution, Biology, Genetics, and Improvement Programs for Eucalyptus globulus and Eucalyptus nitens Around the World. En: $24^{\text {th }}$ Biennial Southern Forest Tree Improvement Conference. Orlando, Florida, USA. June 9-12, 1997. 81-95p. 